\title{
Guiding Parallel Array Fusion with Indexed Types
}

\author{
Ben Lippmeier ${ }^{\dagger}$ \\ Manuel M. T. Chakravarty ${ }^{\dagger}$ \\ Computer Science and Engineering \\ University of New South Wales, Australia \\ $\{$ benl,chak,keller $\} @ c s e . u n s w . e d u . a u$
}

\author{
Gabriele Keller $^{\dagger} \quad$ Simon Peyton Jones \\ $\ddagger_{\text {Microsoft Research Ltd }}$ \\ Cambridge, England \\ \{simonpj\}@microsoft.com
}

\begin{abstract}
We present a refined approach to parallel array fusion that uses indexed types to specify the internal representation of each array. Our approach aids the client programmer in reasoning about the performance of their program in terms of the source code. It also makes the intermediate code easier to transform at compile-time, resulting in faster compilation and more reliable runtimes. We demonstrate how our new approach improves both the clarity and performance of several end-user written programs, including a fluid flow solver and an interpolator for volumetric data.
\end{abstract}

Categories and Subject Descriptors D.3.3 [Programming Languages]: Language Constructs and Features-Concurrent programming structures; Polymorphism; Abstract data types

General Terms Languages, Performance

Keywords Arrays, Data parallelism, Haskell

\section{Introduction}

Haskell is a great language for programming with arrays. Arrays are a natural data type for scientific, engineering, and financial computing, where algorithms are often conceptually functional. Applications in these disciplines typically rely on good runtime performance. Hence, to be useful, Haskell array programs need to perform comparably well to hand-written C programs.

Our Repa library for Haskell does just this $[5,8]$ — alas, it turns out that the programmer needs a very detailed knowledge of Repa to gain this level of performance. For example, consider this simple function, written with Repa version 1.0:

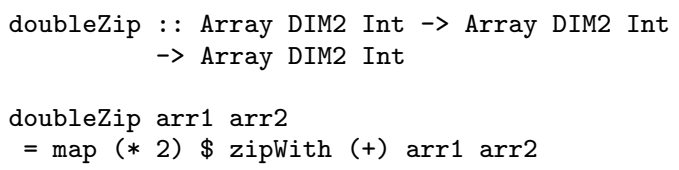

This function appears straightforward, but its performance is awful, especially if its result is used multiple times. To improve performance, users of Repa 1 need to write the following instead:

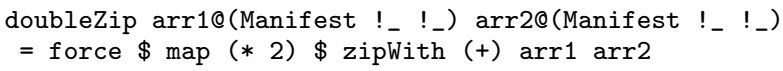

Permission to make digital or hard copies of all or part of this work for personal or classroom use is granted without fee provided that copies are not made or distributed for profit or commercial advantage and that copies bear this notice and the full citation on the first page. To copy otherwise, to republish, to post on servers or to redistribute to lists, requires prior specific permission and/or a fee.

Haskell'12, September 13, 2012, Copenhagen, Denmark.

Copyright (c) 2012 ACM 978-1-4503-1574-6/12/09 . . \$10.00
This second version of doubleZip runs as fast as a hand-written imperative loop. Unfortunately, it is cluttered with explicit pattern matching, bang patterns, and use of the force function. This clutter is needed to guide the compiler towards efficient code, but it obscures the algorithmic meaning of the source program. It also demands a deeper understanding of the compilation method than most users will have, and in the next section, we will see that these changes add an implicit precondition that is not captured in the function signature. The second major version of the library, Repa 2, added support for efficient parallel stencil convolution, but at the same time also increased the level of clutter needed to achieve efficient code [8].

The core idea of the present paper is to replace the hard to understand performance-motivated changes by descriptive types. Here are our main technical contributions:

- We introduce a novel, extensible approach to Repa-style array fusion that uses indexed types to specify the representation and computation methods for arrays $(\S 3)$.

- We show that our use of type indices scales to more elaborate array representations, such as the partitioned and cursored representations [8] $(\S 4)$.

- We compare substantial end-user programs written with the old and new approach, including a fluid flow solver and an interpolator for volumetric data (§5).

- We simplify reasoning about the performance of client programs in terms of the source code while reducing intermediate code size and achieving faster compile times ( $(5)$.

Our improvements are fully implemented and available on Hackage as Repa 3.2.

\section{Representation, Fusion, and Code Explosion}

We start by reviewing the design problems of original Repa library. A simplified version of the core definitions of Repa 1 [5] is in Figure 1. Repa 2 extends the Array type to support more efficient convolutions [8], which we discuss in $\S 2.3$.

Repa 1 introduced delayed arrays to fuse multiple array operations, and minimise the overhead of index-space transforms. Delayed arrays are represented by a function from indices to array elements, as we see in the definition of Array Figure 1. Delayed arrays contrast with manifest arrays, which are represented as contiguous blocks of unboxed values. Fusion of operations on delayed arrays amounts to function composition, as we see in the definition of map. This gives us the map/map fusion rule, map $f \cdot \operatorname{map} g=\operatorname{map}(f \cdot g)$, for free and works similarly for many other operations, including index space transforms such as permutation, replication, slicing, and so on. 


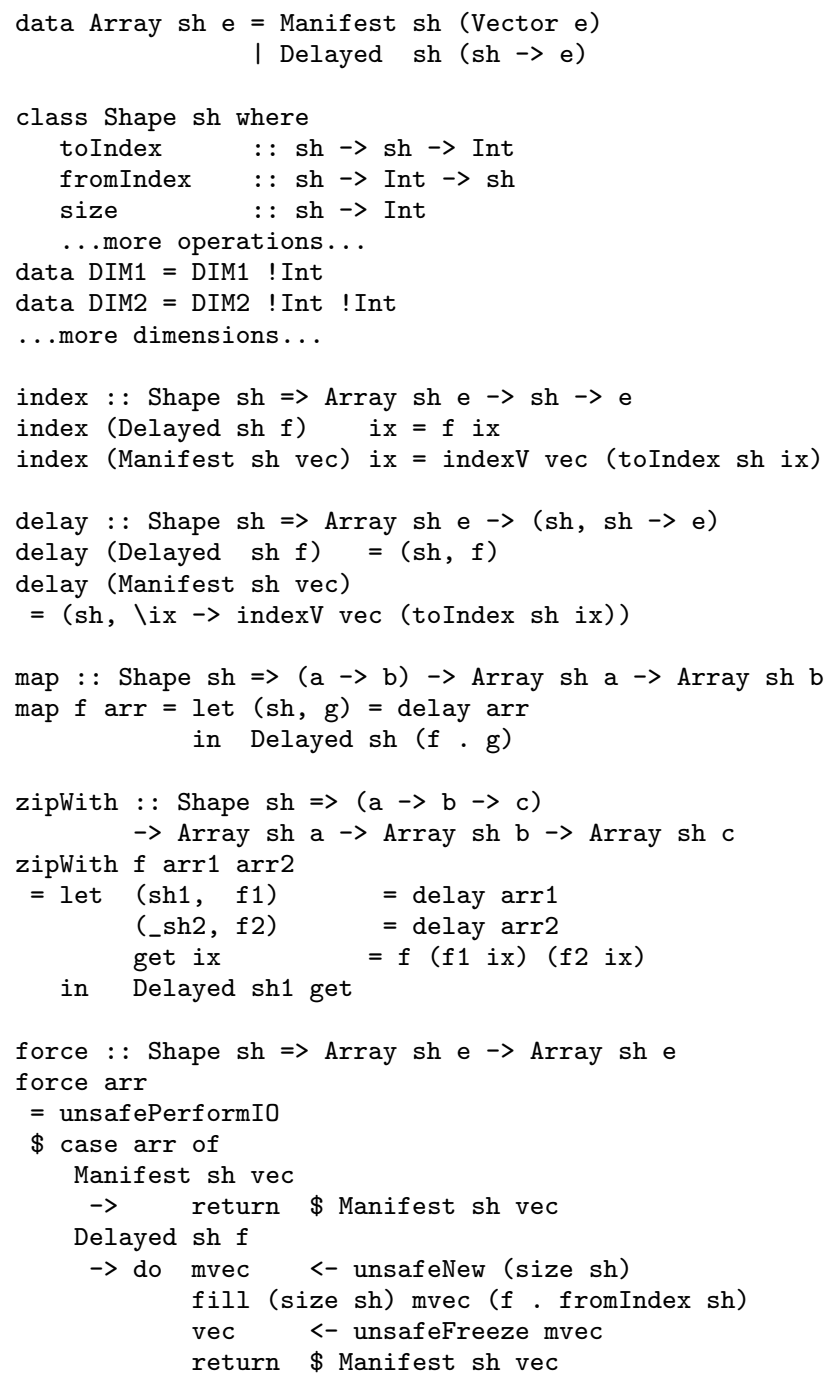

Figure 1. Essential Repa Version 1 Definitions

The elements of a multi-dimensional Manifest array are stored in row-major order in a flat, one-dimensional Vector. The Shape class holds operations to convert between higher-dimensional index types, such as DIM2, and the flat representation. In particular, the toIndex and fromIndex functions convert between higherdimensional and linear indices, and size yields the total number of elements in an array of the given shape. Based on the methods of the Shape class, the function index retrieves a single element from an array, and delay produces an array's shape together with an indexing function to move to the delayed representation. (The function indexV indexes into the flat Vector.)

As stated in the introduction, although Repa $1 \& 2$ can produce efficient code on both sequential and parallel machines [5, 8], they have some significant shortcomings, which we review next.

\subsection{Problem 1: Not Applying force}

To illustrate the problems with Repa 1 , we will reuse the example from the introduction:

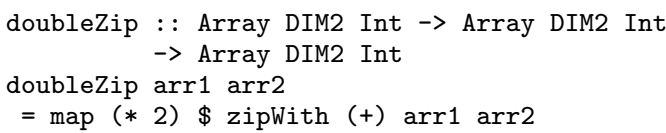

By inlining the definitions from Figure 1 and simplifying, we see that the composition of map and zipWith fuses to produce the following:

$$
\text { let } \begin{aligned}
(\operatorname{sh} 1, f 1) & =\text { delay arr1 } \\
(\text { sh2, f2) } & =\text { delay arr2 } \\
\text { get ix } & =(f 1 \text { ix }+\mathrm{f} 2 \mathrm{ix}) * 2 \\
\text { in Delayed sh1 } & \text { get }
\end{aligned}
$$

The problem is that the array returned by map is not a manifest array, so is not represented as real unboxed data in a contiguous block of memory. Instead, it is a delayed array, represented by a function that takes an array index and computes each element on the fly. The fused code immediately builds a new Delayed array without doing any actual work. This is problematic if the consumer of a delayed array uses elements multiple times. The elements will be recomputed each time, so sharing of results is lost along with runtime performance.

If we desire an array represented by real data, then we should use Repa's force function, which turns a delayed array into a manifest array by executing loop-based parallel array filling code. We would use it in doubleZip as follows:

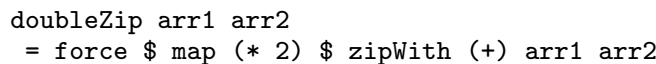

The code here fuses map and zipWith by building a new Delayed array as before. It then fills a freshly-allocated Manifest array, in parallel, using the element-generating function stored in the new Delayed array. In other words, the compiled code will contain an unfolding of the imperative loop provided by force, where the body performs the per-element function, here $(f 1 i x+f 2 i x) * 2$, where $f 1$ and $f 2$ retrieve elements from the two input arrays.

Although our entire approach to parallel array programming hinges on the correct use of force, the type presented in the Repa 1 API documentation was rather uninformative:

force : : Shape $s h \Rightarrow$ Array sh a $\rightarrow$ Array sh a
-- Force an array, so that it becomes Manifest.

From its type alone, force looks like an instance of the identity function. This coupled with the rather cryptic comment, led many users to overlook force entirely. Poor documentation aside, our foundational view that "a type is a name for a set of values" was of no help in expressing the fact that "if you don't use this function your program will be really slow".

\subsection{Problem 2: Runtime Representation Tests}

The version of doubleZip using force produces fused, loopbased code, but is still slower than a straightforward imperative version. This is because the Array type has two data constructors, Delayed and Manifest, so indexing functions must perform a runtime test to distinguish between them. This is a catastrophe if the test is in an inner loop, which is the native environment for indexing functions. In some cases GHC can lift such tests out of loops, but in general such transformations are unsound, because they can change strictness properties if the loop can perform no iterations.

Tantalisingly, the representation of an array at a particular program point does not change from run to run. The programmer always knows which representation is expected - but, in Repa 1 \& 2 they lack a convenient way to express that knowledge. For example, if we know that only manifest arrays will be passed to doubleZip, then we should reify this fact by using explicit pattern matching:

$$
\begin{aligned}
& \text { doubleZip arr1@Manifest }\{\} \text { arr2@Manifest }\{\} \\
& =\text { force } \$ \operatorname{map}(* 2) \$ \text { zipWith }(+) \text { arr1 } \operatorname{arr} 2
\end{aligned}
$$

While this version runs fast, it is awkward due to the implicit precondition: we need to ensure that all callers of doubleZip force the arguments to ensure that they are manifest. 
The test for array representation is not the only run-time test that tends to be needlessly performed in an inner loop. An array also contains size information such as its width and height, which is often used in each iteration. As these are boxed Int values, a loop might repeatedly unbox them, wasting cycles. To ensure the values are unboxed only once, in the preamble of the loop, we need to place a demand on them at the function entry point. We typically do this using bang patterns in the pattern that matches Manifest, and it turns out we also want to demand the flat vector to ensure its components are unboxed as well:

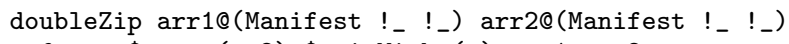

Finally, doublezip runs as fast as a hand-written imperative loop. Unfortunately, the optimisations require reasoning that is not obvious from the source program, demand a deeper understanding of the compilation method than most users will have, and add a precondition that is not captured in the function signature.

\subsection{Problem 3: Inlining and Code Explosion}

In a FORTRAN or $\mathrm{C}$ program, the programmer writes explicit loops. In Repa, the programmer never writes loops; the loops are in library functions. With respect to Figure 1, the key loop is in the definition of fill, which is called by force. The loop code itself is too big to include here, but see [8] for a full definition. The array operations such as map, zipWith and so on, build Delayed arrays by composing functions, but do not contain loops.

How does this turn into efficient code? Consider the last, most efficient version of doubleZip. Inlining zipWith, map, delay, and force, then simplifying yields:

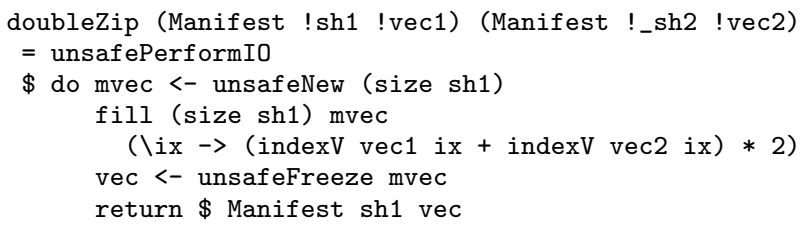

The pattern matching in zipWith's calls to delay are cancelled by the explicitly Manifest arrays; the Delayed array produced by zipWith is canceled by the pattern match in map's use of delay; and so on. When the definition of fill is inlined, we get a tight loop, in which the output is built directly from the input vectors (vec1, vec2) without any intermediates.

Clearly, this fusion depends critically on (a) aggressive inlining and (b) cancellation of statically-visible array construction and pattern matching. However, for efficient stencil convolution, we developed a more complex array representation [8], similar to this:

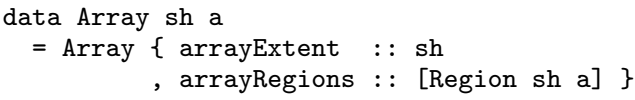

Rather than just being Manifest or Delayed, these arrays consist of a list of rectangular regions. Each region has its own elementgenerating function, which is used to speed up the handling of boundary conditions.

Alas, this representation is fatal for the inline-and-cancel story outlined above. This is because the list arrayRegions must be processed by a recursive function, and compilers (including GHC) are rightly cautious about unrolling recursive functions. In a typical application the programmer knows the exact number of regions at any program point, say four boundaries and a central region. Unrolling five times here would be perfect, but the compiler does not know this.
To make this work, we ended up manually unrolling code in the library functions, by pattern matching on the region list. Here is a typical chunk of Repa 2 library code:

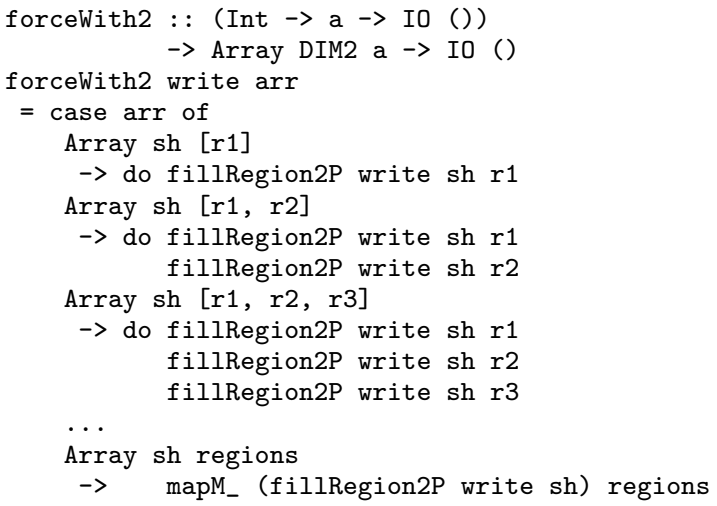

The details are not important, but it should be clear from the form how gruesome this is:

- The library only efficiently accommodates a maximum number of regions. If we use the final alternative of forceWith2 above, then the code will not fuse.

- There is much repetition in the library code.

- The library functions become very large because of the duplication, but they must still be inlined!

Aggressive use of INLINE pragmas produces enormous intermediate programs, which we hope will then shrink radically through construction/pattern-matching cancellation. Sadly, this cancellation does not always happen; imagine that the arr argument of forceWith2 above turned out to be lambda-bound, so that the case remained in the residual program.

\subsection{Summary}

The fundamental problem with Repa $1 \& 2$ is the following: at a particular point in the code, the programmer typically has a clear idea of the array representation they desire. For example, it may consist of three regions, left edge, middle, right edge, each of which is a delayed array. Although this representation is statically known to the programmer, it is invisible in the types and only exposed to the compiler if very aggressive value inlining is used. Moreover, the programmer's typeless reasoning can easily fail, leading to massive performance degradation.

The solution is to expose static information about array representation to Haskell's main static reasoning system; its type system.

\section{The main new idea: Type Indexing}

We are now ready to explain our main technical innovation. In Repa version 3 we define arrays as a data family [2]:

data family Array rep sh e

An Array represents a partial function from indices of type sh to elements of type e. The array is defined on a range of indices, from zero to a maximum called the extent of the array. In this family, rep is a type index that specifies the representation of the array, while $\mathrm{sh}$ is the shape, and e is the element type as before. Figure 2 gives two particular instances of Array, where $\mathrm{D}$ is for delayed and $\mathrm{U}$ is for (unboxed) manifest arrays respectively. 
data family Array rep sh e

data instance Array D sh e = ADelayed sh (sh $\rightarrow$ e)

data instance Array $U$ sh $e=$ AUnboxed sh (Vector e)

...etc...

-- The type indices are declared as nullary types

data D -- Delayed

data U -- Manifest, unboxed

...etc...

Figure 2. Towards the Repa 3 Array Representation

We will give more detail shortly, but we can already see how type indexing addresses the problems of $\S 2$ :

- We can give a more informative type to force:

force :: Shape sh $\Rightarrow$ Array D sh e $\rightarrow$ Array U sh e

Unlike $\S 2.1$, this type statically specifies that the input is delayed and the output is manifest. We cannot accidentally force a manifest array, or forget to force a delayed array in a situation where a manifest one is needed.

- A function like ( $f$ : : Array $U$ sh e $\rightarrow$...) receives an argument that can only be built with AUnboxed. There is no redundant tag-testing to check that the array has the representation that the programer already knows it has $(\S 2.2)$.

- When there are exactly (say) three regions in an array, we can use a type-level list to drive code specialisation, avoiding the ad-hoc approach of $\S 2.3$. Details in $\S 4.2$.

Better still, type indexing scales up to allow a variety of different array representations, each with a different storage/performance cost model. Indeed, Repa 3 has no fewer than ten such representations:

- D - Delayed arrays (delayed) $\S 3.1$

- C - Cursored arrays (delayed) $§ 4.4$

- U - Adaptive unboxed vectors (manifest) $§ 3.1$

- $\mathrm{V}$ - Boxed vectors (manifest) $§ 4.1$

- B - Strict byte arrays (manifest) $\S 4.1$

- F - Foreign memory buffers (manifest) $\S 4.1$

- P - Partitioned arrays (meta) $\S 4.2$

- S - Smallness hints (meta) $\S 5.1 .1$

- I - Interleave hints (meta) $§ 5.2 .1$

- $\mathrm{X}$ - Undefined arrays (meta) $\S 4.2$

We can think of the type indices being generated by this kind declaration:

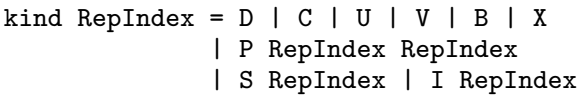

With this declaration, Array ( $P$ U X) sh e is a valid array type. GHC's recent DataKinds extension supports exactly this form of declaration. However, using data kinds would make the index kind closed, preventing users from adding new representation indices. Instead, we proceed as in Figure 2, declaring type indices (such as $\mathrm{D}$ and $\mathrm{U}$ ) as fresh uninhabited types. An open and extensible set of array representations enables integration with other array libraries as the integration with the Accelerate GPGPU library shows. ${ }^{1}$

Of these, the D and C indices specify delayed array representations, meaning the array is expressed as a function from (value)

\footnotetext{
${ }^{1}$ https://github.com/AccelerateHS/accelerate-io
}

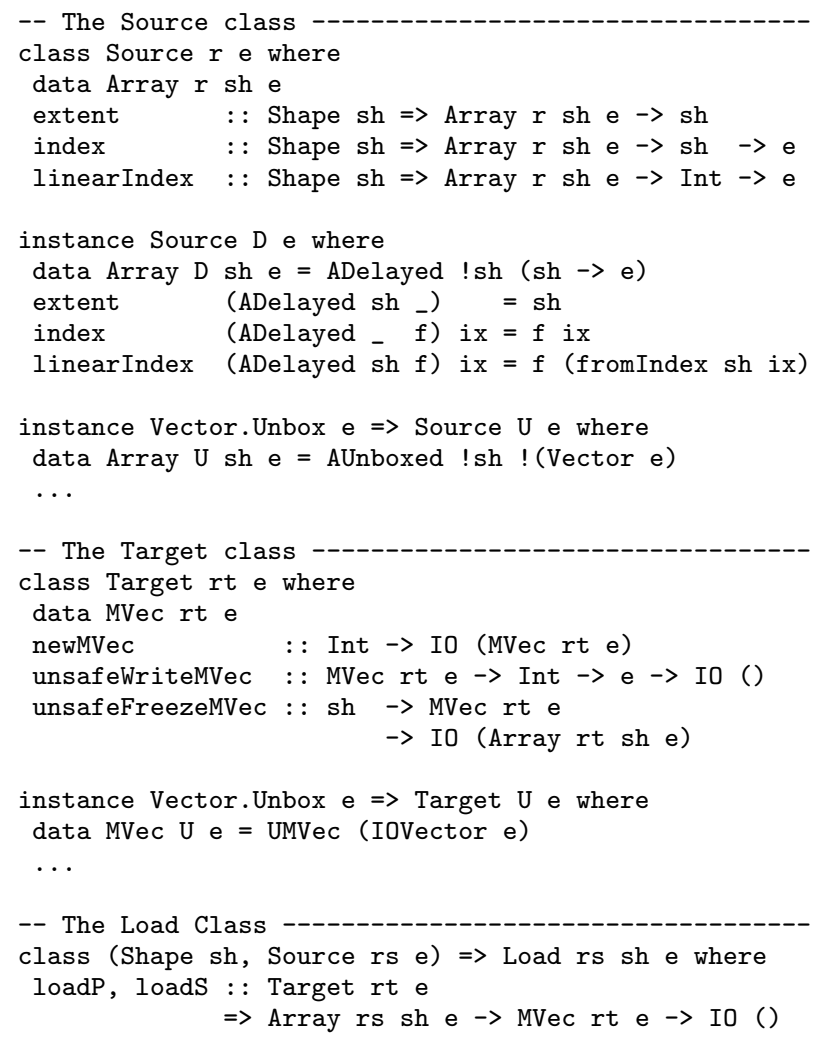

Figure 3. Repa 3 Array Representation

indices to array elements. In this paper we refer to cursored arrays as being "delayed" as well due to the nature of the representation.

The U, V, B and F indices specify manifest representations, meaning real data in memory. Supporting multiple manifest representations makes it easier to interface with third-party array libraries, such as bytestring. The Foreign (F) representation allows us to compute array elements and store them directly into foreign memory buffers, perhaps provided by the operating system. This eliminates copying between the GHC heap and foreign memory that would otherwise be necessary.

Finally, the P, S, I and X indices specify meta representations. They combine other array types or encode information that does not directly define array elements. The partitioned $(\mathrm{P})$ and undefined $(\mathrm{X})$ representations together provide the partitioned arrays from $\S 4.2$. The smallness hint (S) ensures that an array is evaluated sequentially, and the interleave hint (I) manage unbalanced workloads by making each thread compute alternate array elements.

\subsection{Representation-polymorphic Operations}

If we know the type index, we know the array representation, but what if we don't? Fundamental operations, such as array indexing, ought to be polymorphic in the representation. Since the representation of arrays varies with the type index, all polymorphic operations must involve a corresponding type. The canonical approach is to make Array an associated type of a class [2]. The resulting declarations are in Figure 3, which replaces Figure 2.

We see that Array is now an associated type of Source. For each type index (D, U, V, and so on) we define an instance of Source, and each such instance gives a data instance declaration for Array. The methods of Source allow us to perform representation-polymorphic operations on Arrays. The extent 
function takes the shape of an array, and index provides shapepolymorphic indexing. The linearIndex function accesses the underlying flat representation.

The Source class must be parameterised over the representation index $r$ as well as the array element type e, because certain representations restrict the element type. In particular, to store array elements unboxed (type index $U$ ) we need to know (a) the width of the unboxed elements, and (b) the data constructor to use when boxing them. This information is encapsulated in the Unbox class, defined by the standard Vector library, and used in the instance declaration for Source $U$ e in Figure 3.

Note that the Source class contains operations that read or consume an array only. It does not offer operations that construct an array. We keep array-construction methods separate because, in general, they depend on both the source and result representations, and thus require two type indices. We discuss this next.

\subsection{Parallel Computation and Array Filling}

Repa represents manifest arrays by real data in memory. It constructs a manifest array by first allocating a new array, performing parallel writes to initialise the elements, and then freezing the result into an immutable version. These three operations ${ }^{2}$ are bundled into the Target class (Figure 3). The MVec associated type specifies the underlying type of one-dimensional mutable vectors. Delayed and meta representations do not correspond to real data in memory, thus cannot be written to and are not instances of Target.

The Load class, also shown in Figure 3 forms the bridge between Source and Target. The loadP function of Load takes an immutable source array of type Array rs sh e, and a mutable destination vector of type MVec rt e. Instances of loadP will fork several threads to concurrently read elements from the source array, and write them to the destination. The loadS function performs the same operation sequentially, which we discuss further in $\S 5.1 .1$.

With Load and Target, we can write the generic parallel array computation function, computeP, taking the role of force ( $\$ 2.1)$ :

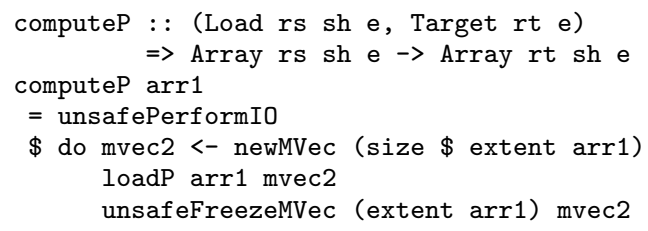

In Repa 3 we use the name computeP instead of force, because the provided Load instances only allow delayed and meta representations to be used as the source. With these representations, loadP runs the delayed computations in parallel. To copy data between manifest representations we provide a separate function copyP which delays the source array before applying computeP. In Repa $1 \& 2$, applying force to an already Manifest array was a no-op. This behaviour turned out to be unnecessary, and needlessly confusing for client programmers.

Finally, we keep the Load and Source classes separate because, for some array representations ( $r s$ ), we want to provide a different loadP instance for each shape (sh). Specifically, the loadP function for DIM2 cursored arrays uses a column-based traversal order, which we discuss further in $\S 5.2 .1$.

\subsection{Bulk Operations}

The definitions of map and zipWith using our new setup are shown in Figure 4. While the bodies of these functions are almost identical

\footnotetext{
${ }^{2}$ The latter two operations have names starting with unsafe because unsafWriteMVec does no bounds checking, and unsafeFreezeMVec does not check that further writes do not take place. This is an internal interface that is not normally used by client programmers.
}

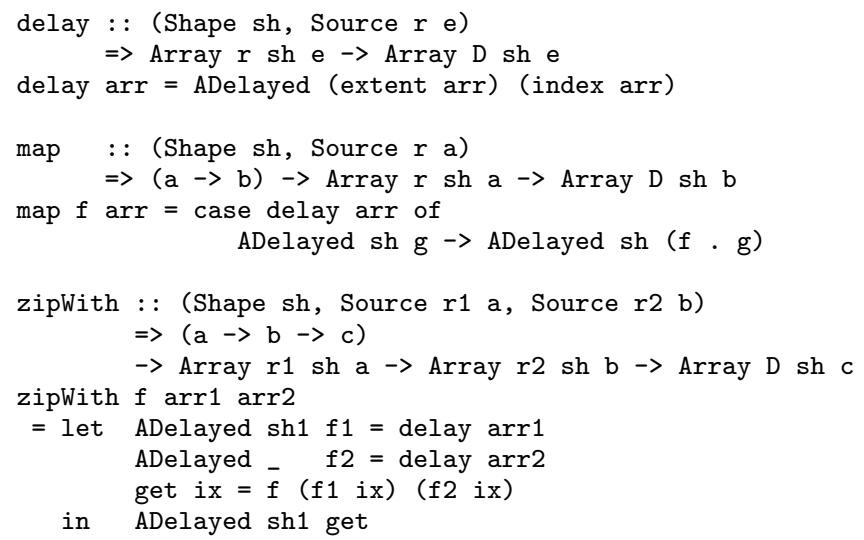

Figure 4. Bulk operations

to the Repa 1 versions from Figure 1, their types now express that the result has the Delayed (D) representation.

Redoing the doubleZip example from $\S 1$ yields:

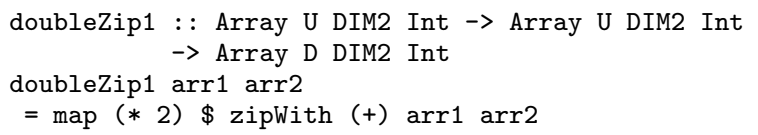

Here we have given a type signature that explicitly constrains the input arrays to have Unboxed $(U)$ representation. The result array must have Delayed (D) representation, corresponding to the signature of map.

When we apply computeP the result array must have a manifest representation, as only manifest representations can be made instances of Target. For example, we could constrain the result to have Unboxed (U) representation as well:

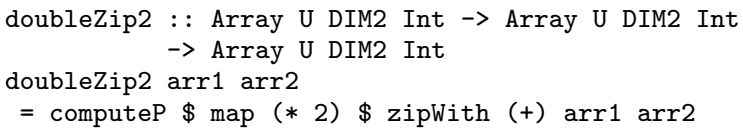

There is no need to provide explicit patterns such as Manifest \{\} for the parameter variables as we did in $\S 1$, because the type index controls the representation directly. Alternately, if we also leave the array shape polymorphic, then the most general type we could assign is the following:

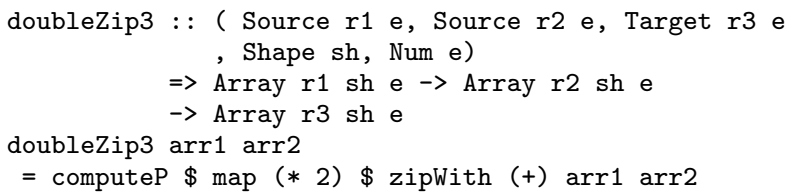

We now return to our stated goal of helping client programmers write fast programs. The fact that an array's representation is determined by its type index provides an easy-to-follow rule specifying when to attach INLINE pragmas to client-written functions:

If the signature contains any D tags, or type class dictionaries, then you must INLINE it to get fast code, otherwise not.

Inlining forces the user-defined element functions in delayed arrays to be fused with applications of computeP present in callers. It also ensures that the instance functions present in type class dictionaries are inlined and fused appropriately. For doublezip2, we do not need to inline it into its caller to get fast code, because the only code fusion that takes place happens within the function itself. 
data F -- Type index for Foreign arrays.

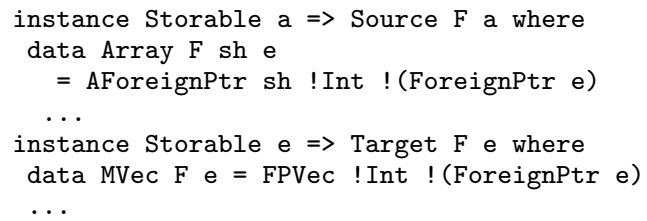

Figure 5. Foreign Arrays

\section{Foreign, Partitioned, and Cursored Arrays}

A major advantage of the type-indexed approach is that it supports a richer variety of array representations, each supporting different usage patterns. In a perfect world one might hope for a single silverbullet representation that magically does everything well. In the messier real world, it is a real step forward to express the cost model clearly but non-intrusively, as we show in this section.

\subsection{Representation Polymorphism and Foreign Arrays}

The code needed to support foreign memory buffers is shown in Figure 5. With this representation we can construct an array directly into a foreign buffer without going via the Haskell heap. This is achieved with the following function:

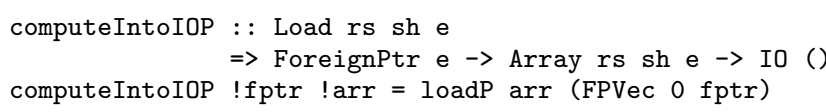

Rather than returning the manifest array like computeP, this function takes the address of a foreign buffer and fills it by side effect in the IO monad.

\subsection{Partitioned Arrays}

A partitioned array consists of two neighbouring rectangular regions, each represented by their own Array. If these arrays are themselves partitioned, we can sub-divide an array into any number of regions. Our primary use-case is to represent the result of a stencil convolution, where the element function that defines the inner region does not need to worry about what to do when applied close to the border of the array [8].

The representation of partitioned arrays is shown in Figure 6. The data declaration for Array ( $\mathrm{P} r 1 \mathrm{r} 2$ ) defines a partitioned array to consist of two sub-arrays (apHead and apTail), together with a field, apHeadRange, that defines the range of indices covered by apHead. Somewhat irregularly, the sub-arrays are indexed directly by the index of the outermost array, so the sub-arrays cover an index range that may not be zero-based. To index an element in a partitioned array, we use the rangeMatch field of apHeadRange to test whether its index is within the range of apHead and if so index into arr1, otherwise we index into arr2. The Range type defines a rectangular range of elements between the two indices with type sh, and our Load instance uses these two fields to compute starting and ending offsets during parallel computation:

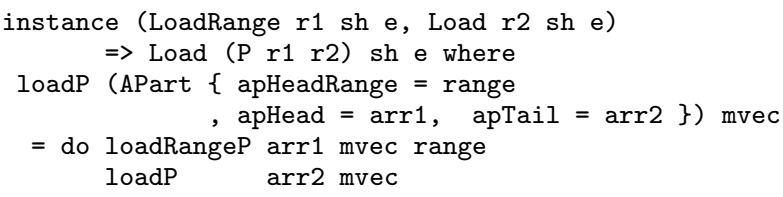

The Load class declaration was given in Figure 3. The above instance uses the auxiliary class LoadRange (Figure 6), which is just like Load, except that it only computes and writes elements in a specified Range. Since apHeadRange only describes apHead,

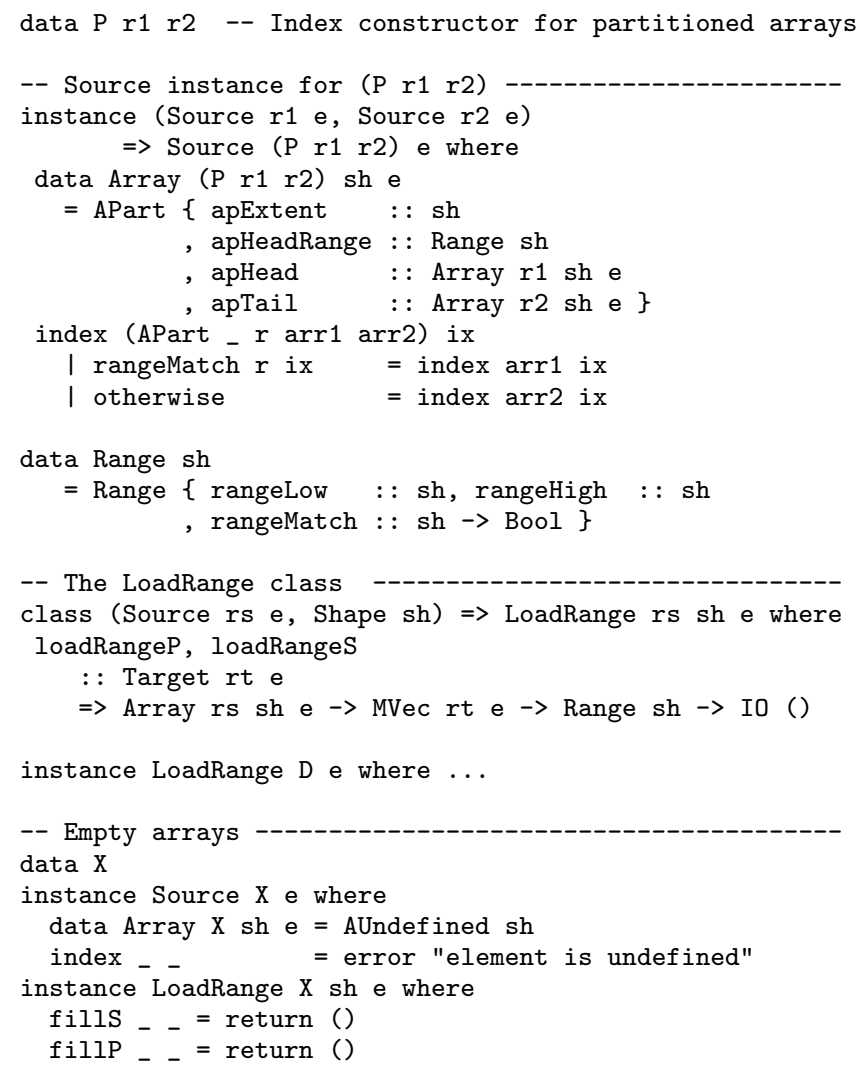

Figure 6. Partitioned Arrays

we use loadRangeP for apHead, and loadP for apTail. When we reach the right-most array (at the end of the apTail chain) we have no explicit description of the range of indices covered. In this case we use an empty array with type index X (see Figure 6). Thus a typical partitioned array might have a type like PD5 in Figure 8; note that X terminates this "list" of partitions.

However, the critical point is this: the loadP instance for partitioned arrays can be completely unfolded by the GHC simplifier at compile-time. For example, given the following call:

loadP (arr : : Array (P D (P D X)) DIM2 Float)

GHC can inline the code for loadP at type ( $P$ D ( $P$ D X)), which produces code with a call to loadP at type ( $\mathrm{P}$ D X). GHC can inline that too, yielding code that calls loadP at type $\mathrm{X}$, and that can be inlined trivially. The result is a sequence of two calls to loadRangeP, each at type D:

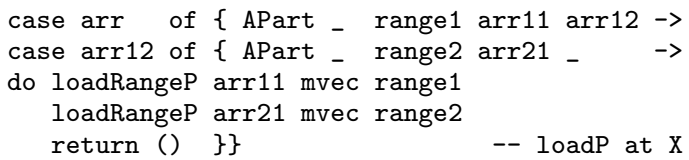

Now GHC can inline the two calls to loadRangeP at type D. We end up with a sequence of two loops, each executed in parallel.

This kind of inlining is guaranteed not to diverge, because the type of arr 12 becomes smaller in each recursive call, providing a structural termination condition for loadP. This is similar to the termination conditions used by theorem proving languages such as Coq [3] and Agda [10]. The use of type indices to guide the compiler solves the code explosion problem discussed in $\S 2.3$, as our array filling functions are now unfolded only as many times as needed for the source array. 


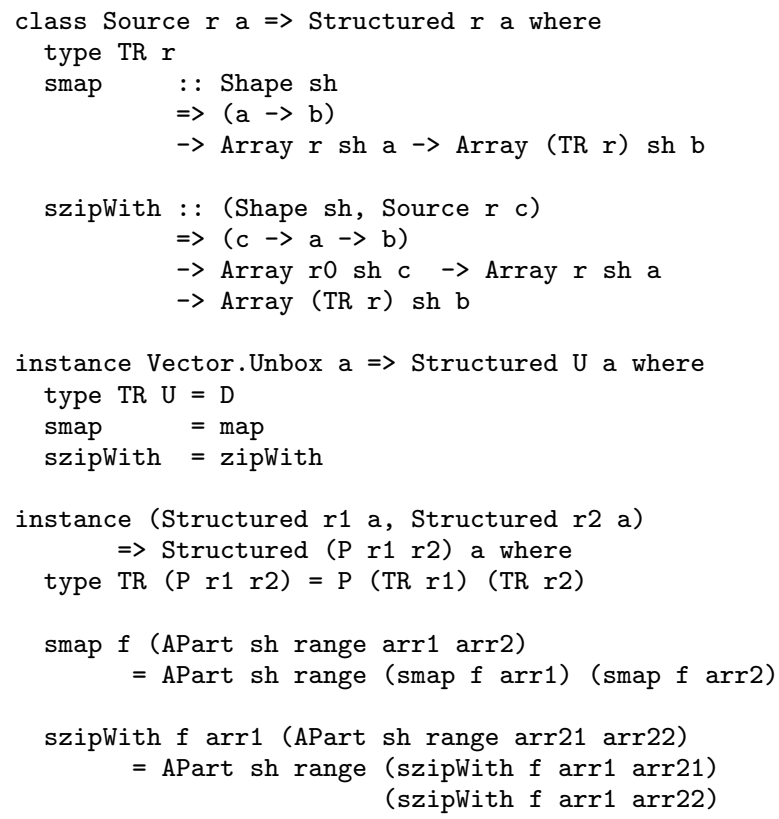

Figure 7. Structured Maps

\subsection{Structure Preserving Maps}

Say we have arr : : Array (P D (P D X)) DIM2 Float. As we saw at the end of $\S 4.2$, the application loadP arr will compile to two beautiful loops, one for each partition. However, suppose we also map a function across every element before loading from it, like with (loadP (map negate arr)). Referring to Figure 4, we see that map always produces a delayed result, with type index D. The loadP only sees a delayed array, and will generate a single loop in which each indexing operation performs a conditional test on arr to determine which partition to use. Disaster: this is slower than not having partitioned arrays in the first place.

What we want is for map to be structure-preserving: given a partitioned array, it should produce a partitioned array. However map should not always produce an array with the same representation as its input. Given a manifest array, map should produce a delayed array. In short, the appropriate representation of map's result is a function of the representation of its input. This is just what type functions are for!

Figure 7 implements this idea. We use a new class Structured, whose methods are smap and szipWith. The class has an associated type TR (short for Target Representation), which computes the result representation from its argument. We can see a use of TR in the type of smap.

The U instance of Structured is simple, we just use the default map implementation from Figure 4. The ( $P$ r1 r2) instance from Figure 7 is more interesting, as it preserves the partitioning structure of the source array.

Continuing on to szipWith, note that its type is right biased. The structure of its result is taken from the structure of the second array argument, ignoring that of the first. Preserving the partitioning of both source arrays would be significantly more complicated. For example:

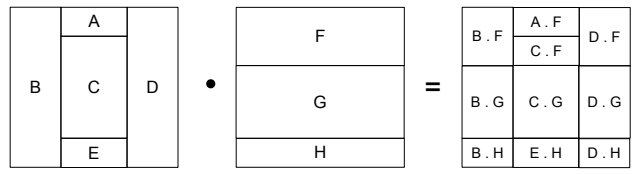

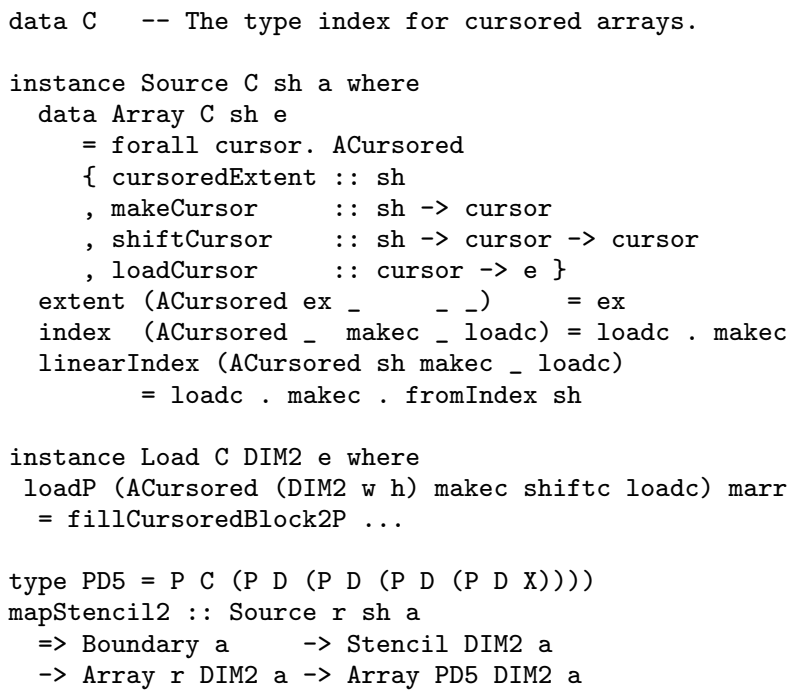

Figure 8. Cursored Arrays

The number of partitions in the result depends on the number of partitions in the input arrays, which is a static property, as well as the sizes of those partitions, which can be a dynamic property.

Repa 3 includes the original zipWith as well as our new szipWith function. With plain zipWith, if the overall shape of the source arrays is different, then the shape of the result is their intersection. Performing this intersection is straightforward when there is no internal structure to worry about. In contrast, szipWith preserves the structure of the second source array, but the overall shape of both source arrays must match.

\subsection{Cursored Arrays}

The cursored arrays of [8] are used to optimise stencil convolutions, by sharing intermediate values between the computation of adjacent pixels. Figure 8 contains the definition of cursored arrays using our new type-indexed framework. The Array declaration in Figure 8 takes the role of the Generator from Repa 2, with the role of makeCursor, shiftCursor and so on being as per [8].

The definition of fillCursoredBlock2P in the Load instance of Figure 8 is as per [8]. As discussed there, its definition contains loops that have been hand-specialised with the unroll-andjam transformation [1] to separate array reads from array writes. This in turn enables LLVM's global value numbering transformation [14], which recovers sharing of intermediate results between the computation of successive array elements. To improve cache performance, these loops also traverse the source and result arrays in column-wise order, as per the diagram in $\S 5.2 .1$. This means that fillCursoredBlock2P is specialised for arrays of rank-2, hence the DIM2 constraint in the Load instance it is used in.

The mapStencil2 function takes a stencil definition, a source array, a description of what to do at the boundary; and produces a partitioned array. This function is also specialised to rank-2 arrays, so the result is split into five partitions, one for inner region and one for each of the four borders. As the use of cursored arrays tends to increase the size of the intermediate code due to loop unrolling, we used a Cursored (C) array for the inner region only, defining the borders in terms of Delayed (D) arrays. The runtime cost of computing the border regions is typically only a tiny fraction of the cost of computing the internal region, and using delayed arrays for the borders keeps the compile-times and resulting executable size down. 


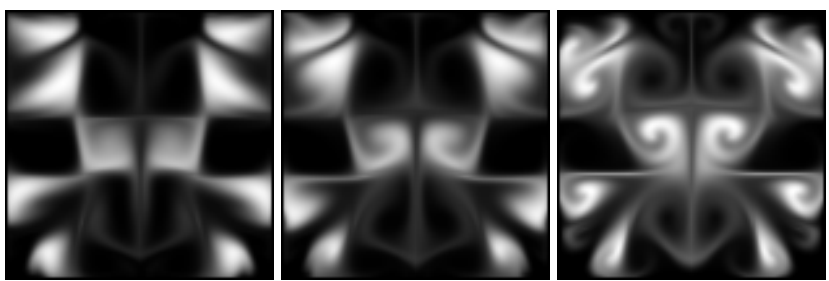

Figure 9. Fluid Solver output for 4, 10, and 100 Jacobi iterations.

\section{Applications and Pragmatics}

In this section we discuss two end-user applications that were first written with Repa 2, and then modified to work with Repa 3 by the first author. Providing a better way to implement these applications was the reason we started this paper. The first is available in the gloss-examples package, and the second in the repa-examples package. Gloss is a graphics library that depends on Repa.

\subsection{Fluid Flow}

Figure 9 shows output from a fluid flow solver written by Ben Lambert-Smith. This is an implementation of Jos Stam's stable fluid algorithm [18], which is a fast approximate algorithm intended for animation and games, rather than accurate engineering simulation. It performs a finite time-step simulation on a 2-d grid. It is numerically stable for arbitrary sized time-steps, which makes it attractive for real-time animation where the frame-rate of the simulation may vary depending on system load. We discuss the difference between the three images of Figure 9 in $\S 5.1 .2$.

The fluid is represented as a pair of 2-d arrays, one for the density at each point, and one for the velocity. The density is a scalar floating point value, while the velocity is a 2 -d vector. The majority of the runtime is spent in a function called the linear solver, which performs matrix relaxation involving the discrete Laplace operator $\left(\nabla^{2}\right)$. The linear solver is used to diffuse the density and velocity fields throughout the grid, as well as apply a projection operator to the velocity field, which makes it masspreserving [18].

Our implementation of the linear solver uses Repa's support for stencil convolution, using the cursored arrays from $\S 4.4$, and repeatedly computes the following for each grid element:

$$
u_{i, j}^{\prime \prime}=\left(u_{i, j}+a \cdot\left(u_{i-1, j}^{\prime}+u_{i+1, j}^{\prime}+u_{i, j-1}^{\prime}+u_{i, j+1}^{\prime}\right)\right) / c
$$

For each time step we perform several relaxation iterations to allow the solution to converge. In the above equation, $u$ is the grid in the previous time step, $u^{\prime}$ is the grid in the current time step and the previous relaxation iteration, and $u^{\prime \prime}$ is the grid in the current time step and current iteration. The $a$ and $c$ values are constants determined by simulation parameters, such as the diffusion rate.

The linear solver is written to be polymorphic in the type of array elements. It is then specialised to 2 -d vectors (for the velocity) as well as scalar floats (for the density) using GHC's specialisation pragmas [12].

\subsubsection{Scheduling and Smallness Hints}

In the Repa version, the linear solver is called four times: once on the scalar density field, and three times on the vector velocity field. Each time it is called, it iteratively performs 40 convolutions with the appropriate stencil, for a total of 160 convolutions per time-step. As the algorithm is intended for real-time simulation, at 30 frames (steps) per second it must perform $30 \times 160=4800$ convolutions per second, or one every 200 microseconds. This interval is of the same order of magnitude as the context switch latency on a typical desktop operating system [7].
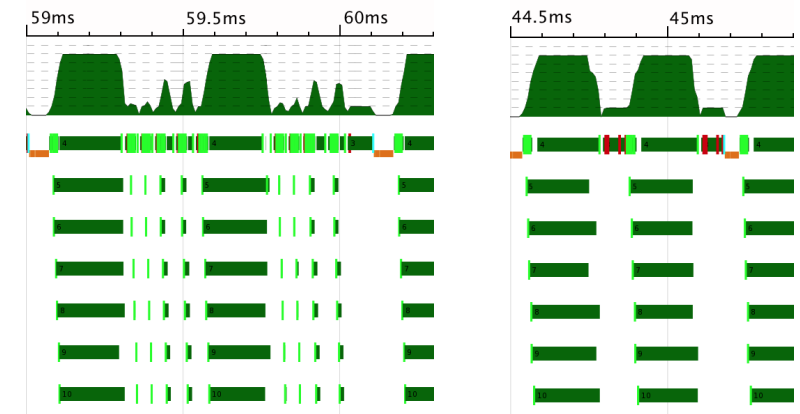

Figure 10. Fluid Thread Activity

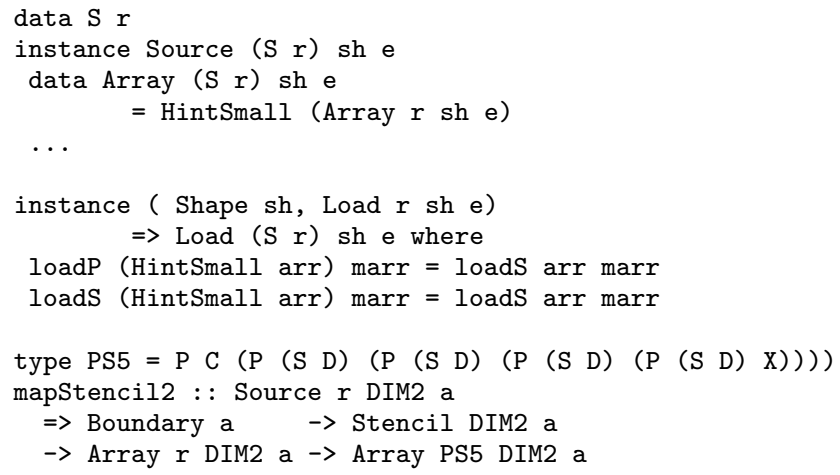

Figure 11. Smallness Hints

When benchmarking the fluid solver using ThreadScope [4], we noticed that for a grid of size $100 \times 100$ it was spending over half its time stalled while waiting for worker threads to be scheduled. For context, with a single thread, a grid of size $150 \times 150$ is about largest that will run smoothly at 30 frames per second on our desktop machine. We present concrete numbers in Figure 12.

The left of Figure 10 is a ThreadScope plot for the $100 \times$ 100 version showing the problem. This plot was taken on an $2 \times$ QuadCore Intel Harpertown server running Linux Kernel 2.6.32. To minimise the chance of our benchmark being interrupted by the operating system, it was run with only 7 threads (out of 8 possible), leaving the final core for the OS. We use thread affinity to bind each Haskell thread to a single core. In the figure, the 7 horizontal traces show the period each thread was active. The graph at the top shows how many threads were active at each point in time.

The graph shows two bursts of high activity where the benchmark was performing a matrix relaxation step, and the start of a third one on the far right hand side. As mentioned in $\S 5.1$, the matrix relaxation is performed using a stencil convolution, which uses the partitioned array representation from $\S 4.2$. Each burst of high activity, where the plot shows all seven threads active, corresponds to the computation of the inner region of the array. The four short bursts after it correspond to the computation of the border regions. Because computation of a border region is so little work, more time is spent waiting for the computation to be scheduled than actually computing it.

We address this problem with smallness hints, which are wrappers for Repa's usual Delayed (D) and Cursored (C) type indices. The definition is given in Figure 11. Whereas the application of computeP from $\S 4.2$ to an array of type Array D DIM2 Int will proceed in parallel, application to an Array (S D) DIM2 Int will proceed sequentially. This behaviour is straightforward to add 
to our existing framework, as the evaluation method for each array representation is given by the corresponding instance of the Load class. Given some inner representation $r$, the Load instance for $\mathrm{S} r$ simply redirects applications of both loadP and loadS to the loadS method for $r$.

We force the borders of a partitioned array to be evaluated sequentially by modifying the definition of mapstencil2 from $\S 4.2$. All that is needed is to wrap the existing border definition in the HintSmall constructor. The effect on the type of mapStencil2 is also shown in Figure 11.

The ThreadScope plot in the right of Figure 10 is for the same benchmark, now using smallness hints for the border partitions. Now only the main thread is active between each high-activity burst, yet the period of low activity is shorter.

There is a design choice about whether to preserve smallness hints in the result of an smap operation $\S 4.3$. Although computation of a particular region in a delayed array may correspond to a small amount of work, after we map a function across every element, computation of the same region in the result may be more expensive. For now we arrange smap to preserve the smallness hint in the result array, though we will return to this in $§ 5.2 .2$.

\subsubsection{Gauss-Seidel vs Jacobi relaxation}

The reference C implementation of Jos Stam's fluid flow algorithm was supplied with [18]. The linear solver in this version uses GaussSeidel matrix relaxation while we use Jacobi relaxation. Relative to the equation in $\S 5.1$, Gauss-Seidel relaxation replaces the $u_{i-1, j}^{\prime}$ and $u_{i, j-1}^{\prime}$ terms with $u_{i-1, j}^{\prime \prime}$ and $u_{i, j-1}^{\prime \prime}$ respectively. In the reference version these array elements are read from the array currently being written to. Jacobi relaxation uses the equation as written.

Although the "fast forwarding" of array elements in GaussSeidel reduces the number of iterations needed to achieve convergence, its use of destructive update makes it difficult to parallelise. Destructive update also causes problems for optimising compilers such as LLVM (which GHC uses for back-end compilation) as they must worry about potential aliasing between the source and result arrays. In contrast, Jacobi relaxation is kinder to optimising compilers, and easier to parallelise, but requires more iterations than Gauss-Seidel as it does not converge as fast.

For Stam's algorithm, the penalty for using an insufficient number of iterations is an unattractive image with too much numerical dissipation [18]. Figure 9 shows the result of simulating 100 time steps from identical initial conditions, using 4, 10 and 100 Jacobi iterations in the linear solver. For low iteration counts, the swirls present in the right-most image do not appear in the output.

To ensure a fair comparison, our Repa implementation using Jacobi relaxation must use more iterations than the reference implementation. We determined an appropriate number by first simulating the initial conditions used in Figure 9 using 1000 Gauss-Seidel iterations, treating this as the ideal output. We then measured the mean-square error between the ideal output, and the output using 20 Gauss-Seidel iterations, which is what Stam's reference implementation uses. Finally, we increased the number of Jacobi iterations until the error in the output was reduced to the same level as the reference version. Using 38 Jacobi iterations achieves the same error figure, which we round up to 40 for good measure.

\subsubsection{Comparison}

Figure 12 shows the relative runtimes between Stam's C implementation using Gauss-Seidel relaxation (with 20 iterations), and the same program modified to use Jacobi relaxation (with 40 iterations). We also show relative runtime for the Repa version using Jacobi relaxation with 1, 2 and 4 threads.

The overall shape of this plot is as we would expect. At small array sizes the sequential $\mathrm{C}$ versions are faster as they preallocate

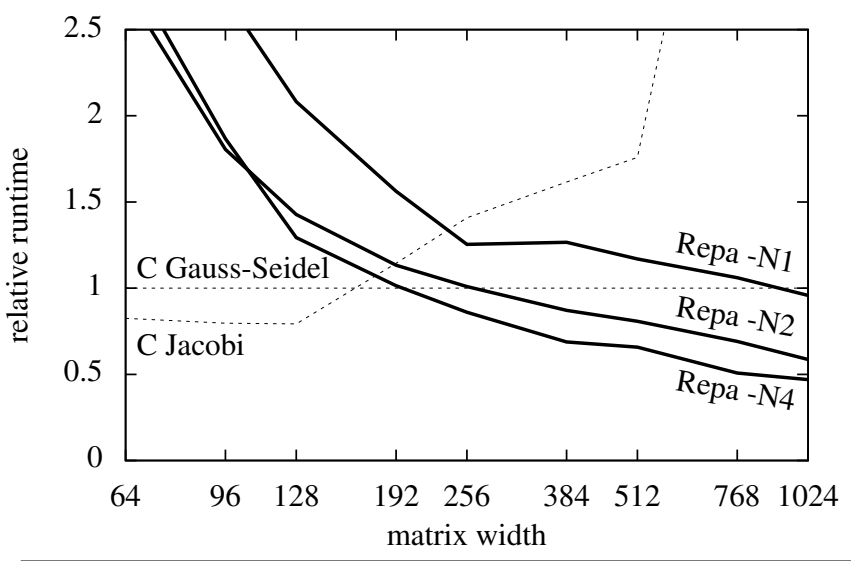

Figure 12. Runtimes for Fluid Flow Solver

buffers for the source and result arrays, and swap them after every iteration. This improves data locality, reducing the cache miss rate. In contrast, the Repa version allocates a fresh result buffer for every iteration, leaving old buffers to be reclaimed by the garbage collector.

At large array sizes, the working set no longer fits in cache and the single threaded Repa Jacobi solver is faster than the $\mathrm{C}$ version. This is because cursored arrays allows the Repa version to share intermediate computations, which reduces overall instruction count and memory bandwidth. For large array sizes the benchmark is memory bound, so performance does not scale linearly with an increased number of threads.

The $\mathrm{C}$ reference implementation could be improved by handapplying the unroll-and-jam transformation that is baked into the Repa library. We tried various permutations of -funroll-loops when compiling with GCC 4.2.1, but inspection of the assembly output revealed it was not recovering the same inter-stencil sharing as the Repa version due to aliasing problems - even though the loops were indeed unrolled. Compiling with Clang 3.0 (which uses LLVM for the backend) did not significantly improve matters. On the other hand, we could also improve the Repa version by preallocating the source and result arrays and using the ForeignPtr support to swap and reuse the same buffers between iterations.

\subsection{Unbalanced Workloads}

Figure 13 shows three example applications with unbalanced workloads, all written with Repa. The first is a Mandelbrot set visualisation computed with the escape-time algorithm. In the output image, the pixels in the (approximate) Mandelbrot set are rendered black and take about 10 times longer to compute than the others.

The second is output from a real-time ray tracer, where parts of the image showing many reflections take longer to compute than the others. Although ray tracing is known in the folklore as "embarassingly parallel" as every pixel in the output can be computed independently, it is not embarrassingly data parallel due to the unbalanced workload.

The final example is an interpolator for volumetric data, which implements the algorithm described in [17]. This example was written by Michael Orlitzky using Repa 2, and then modified to work with Repa 3 by the first author. The left-most image at the bottom of Figure 13 shows one slice though a $256 \times 256 \times 109 \times 16$-bit data volume from a Magnetic Resonance Imaging (MRI) machine. The bottom-center image is from the source data, and shows a scaled region of the top-right portion of the brain. The bottom-right image shows the same region after interpolation. In a straightforward 


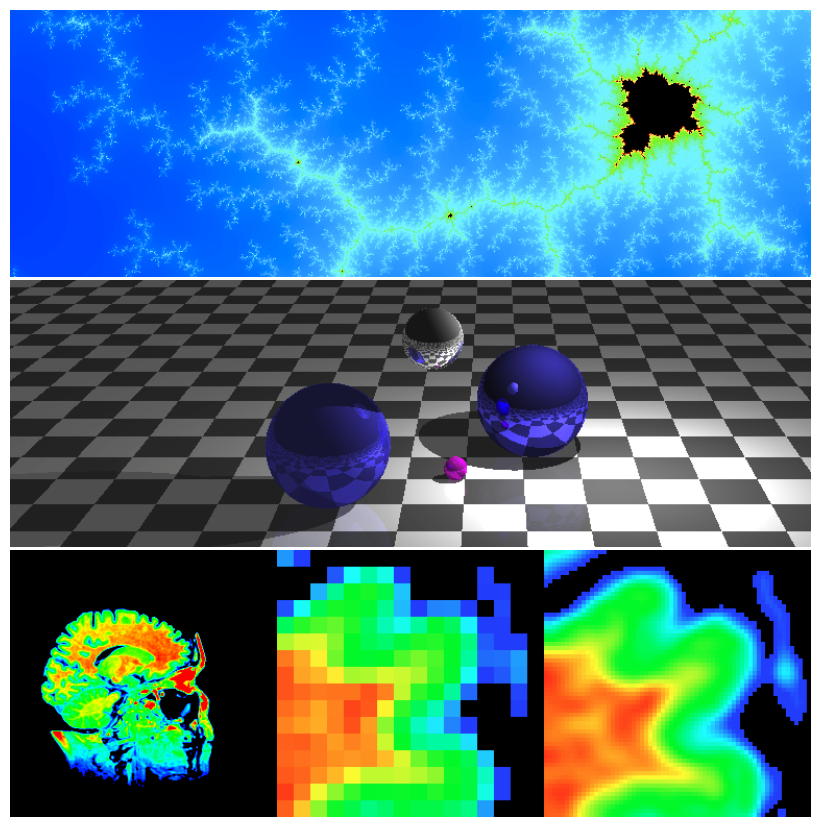

Figure 13. Unbalanced Workloads

implementation, every element in the output volume is computed independently and takes the same amount of time. However, we can improve the overall runtime by returning a constant zero value (black pixel) for voxels corresponding to the air surrounding the physical object. This is done by summing the surrounding voxels in the source data, and testing the sum against a user defined threshold. This is faster than calculating the true interpolated result, but again makes the workload unbalanced.

\subsubsection{Spacial Correlation and Interleaved Evaluation}

The workloads of our three examples are unbalanced because the cost to compute each array element is not uniform throughout the array. The standard Repa evaluation method chunks the underlying row-major vector evenly between the available threads. When using cursored arrays we instead proceed column-wise as this is more cache-efficient when performing convolutions on 2-d matrices. The figure below shows both of these methods, assuming we are computing the matrix with three threads.

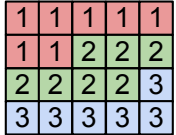

Chunked

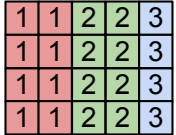

Column-wise

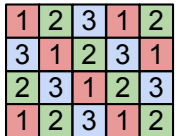

Interleaved
With both the Chunked and Column-wise method, the spacial correlation between features in the result array, and computational workloads maps directly onto the physical processors. The left of Figure 14 is a ThreadScope plot that shows the effect of this correlation in sharp relief. The plot is for the interpolator on seven threads, which shows the threads that compute non-zero data in the result take significantly longer to run. The plot is for the entire run of the program, and the high-activity bursts at the beginning and end are due to reading source data and writing the output to file.

A well known solution to this problem is to move to an interleaved evaluation method instead [15], also shown in the figure above. When applied to ray tracing this approach is classically
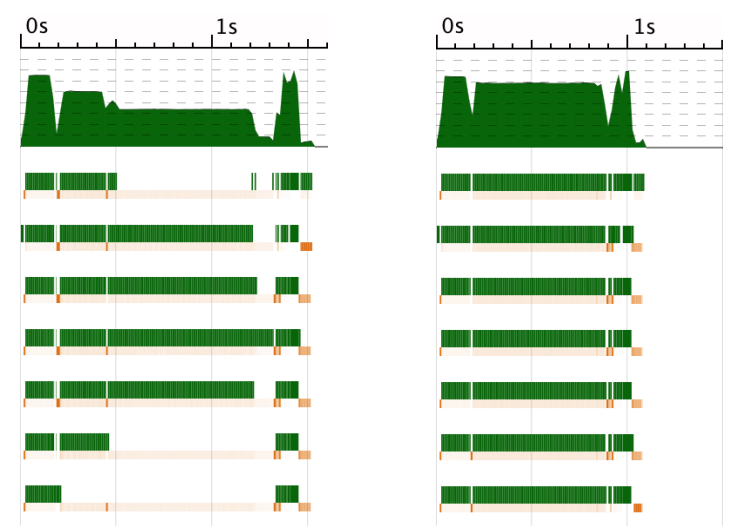

Figure 14. Interpolator Thread Activity

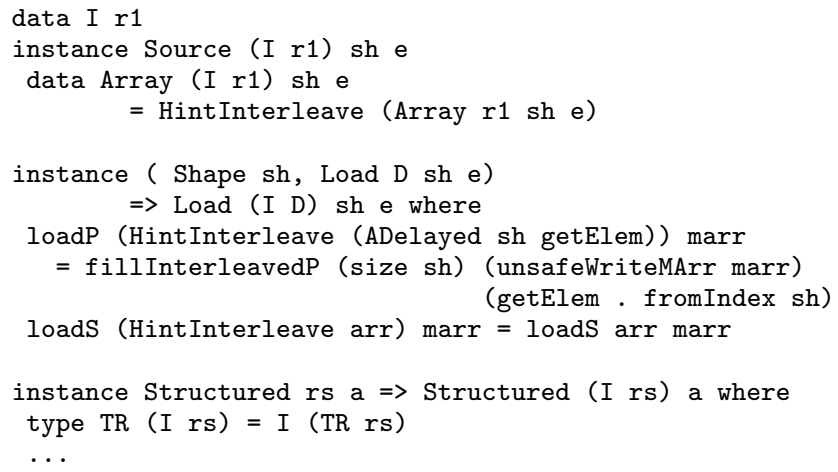

Figure 15. Interleave Hints

known as image space partitioning to distinguish it from object space partitioning which divides the model being rendered. As with all static load-balancing strategies, there is still a chance that the runtime-workload will correlate with the assigned thread index, though this would be unlikely for the three applications shown in Figure 13. Lee and Raghavendra [6] compare related strategies.

We implement our new interleaved evaluation method similarly to the smallness hints from $\S 5.1 .1$, with the main definitions given Figure 15. Whereas application of computeP to an array of type Array D DIM2 Int will use chunked evaluation, application to an Array (I D) DIM2 Int now uses interleaved evaluation, implemented by fillinterleavedP. The right of Figure 14 shows the result of using interleaved evaluation for the interpolator. All threads now run for approximately the same period of time, and of the overall runtime of the program is shorter.

\subsubsection{Hint Propagation and Interaction}

The Load instance in Figure 15 only works for Delayed (D) arrays, and not Cursored (C) arrays as well. As described in $\S 4.4$, cursored arrays are used to share intermediate computations between adjacent array elements, and this process depends on a particular traversal order. As adjacent elements must be computed in the same loop iteration, using interleaved evaluation with cursored arrays would be of no benefit.

Smallness hints and interleave hints interact in a natural way. If a delayed array is wrapped in an Interleave (I) hint, this signals that its parallel computation will be unbalanced. If it is then wrapped in a Smallness (S) hint as well, this signals that it is a small amount of work relative to some larger computation. The combination of 
hints yields a type index of ( $S$ (I D ) ). When the array is finally computed, the instances given in Figures 11 and 15 effectively ignore the interleave hint, as the sequential evaluation enforced by smallness cannot itself be unbalanced. If the two hints are applied in the other order, to yield an index of ( $I$ (S D)) then there is no available Load instance, because hinting that a sequential computation is unbalanced does not make sense.

Finally, note that the Structured instance in Figure 15 propagates the interleave hint to the result representation. The declaration of Structured was given in Figure 7. We preserve this hint because the Structured class methods, namely smap and szipWith are bulk operations, meaning they apply the same function to every array element. In practice, it is highly unlikely that applying such an operation to an array defining an unbalanced workload would make it more balanced, so it is better to retain the unbalancedness. For example, suppose we apply our ray-tracer to a $3 \mathrm{~d}$ model and then convert the output image to greyscale:

image : : Array U DIM2 Float

image $=$ computeP $\$$ smap toGreyScale $\$$ raytrace model

The result of evaluating raytrace model will have the type Array (I D) DIM2 (Float, Float, Float), where the tuple contains red, green, blue color values. Applying smap toGreyScale the produces Array (I D) DIM2 Float, where the result gives a luminance value for each pixel. The array defined by the raytrace is unbalanced, and when fused with toGreyScale it remains unbalanced.

\section{Challenges of Array Fusion}

This section summaries the remaining challenges we see to the Repa-style approach to array fusion. We continue the similarly named section in [8].

\subsection{Unboxing Outside Loops}

In [8] we used boilerplate code involving deepSeqArray to force GHC to unbox array objects outside of loops. Adding this code worked around limitations in the simplifier for GHC's core IR. For example, consider the following function which takes an array of indices, a matrix and yields elements from the matrix diagonal:

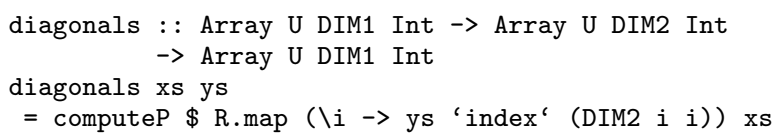

As the ys array is only used inside the worker function passed to map, with lazy evaluation this array will only be demanded if xs contains at least one element. As the GHC simplifier mostly ${ }^{3}$ tries to preserve the termination behaviour of the program during transformation, it does not float this unboxing out of the loop. It must guard against the case where evaluation of ys diverges, hence the components of ys end up being unboxed repeatedly for every iteration. Even with pointer tagging [9], the cost of unboxing values in inner loops can easily dominate the runtime of an array program.

With Repa 2 and GHC 7.2 we needed to use deepSeqArray to place a demand on the components of ys, to ensure their unboxings are floated outside the loop:

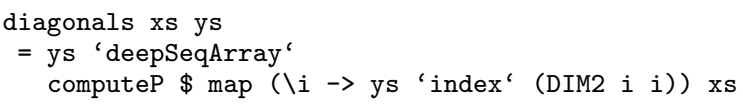

In GHC 7.4.1 we implemented case-floating. This transform operates much like let-floating [11], except that it moves singlealternative case expressions.

\footnotetext{
${ }^{3}$ In GHC 7.4.1, non-termination is not preserved by eta expansion, but correct termination behaviour can be gained with -fpedantic-bottoms.
}

With case-floating, instead of needing deepSeqArray we can achieve fast code by using a lighter-weight bang pattern:

diagonals $\mathrm{xs}$ !ys $=\ldots$

This then desugars to:

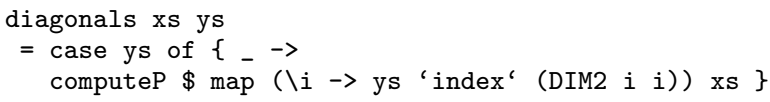

When the definition of index is inlined we get:

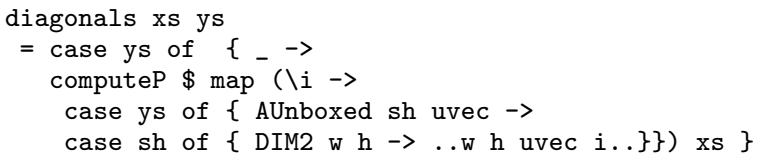

Now, as ys is demanded on entry to the function, the inner match against AUnboxed sh uvec can be unconditionally moved to top level. However, hoisting the match against DIM2 $\mathrm{w} \mathrm{h}$ is only sound when the shape of an array is defined as a strict field, though from Figure 3 we see that it is. Moving the match on DIM2 $\mathrm{w} h$ to the outer case expression based on this strictness information is our new case-floating transform:

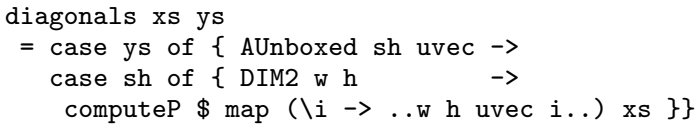

In practice, we advise users to add bang patterns to all array parameters for functions using the Repa library. Although the xs parameter above does not need one, adding it does not hurt, and this is an easy-to-follow rule.

Sadly, bang patterns are not always sufficient. Suppose ys is defined as a top-level CAF:

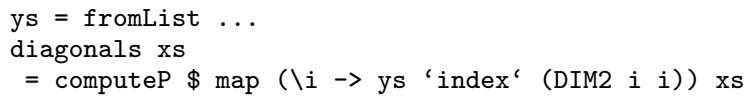

In this situation the language definition does not allow us to place a bang on ys. This would imply that ys should be evaluated as soon as the program starts, which is problematic if it happened not to terminate. Instead we add a seq, like so:

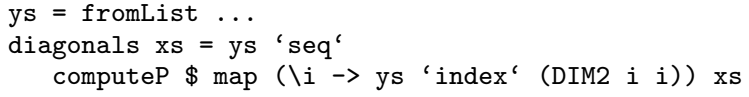

The seq desugars to a case-expression as above. The fact that seqs must still be added to get efficient code is not kind to beginning Haskell programmers, but we do not see a way to avoid it with the current language semantics.

\subsection{Fake Nested Parallelism via Laziness}

The following example is like diagonals from the previous subsection, except that it first increments every element in the matrix.

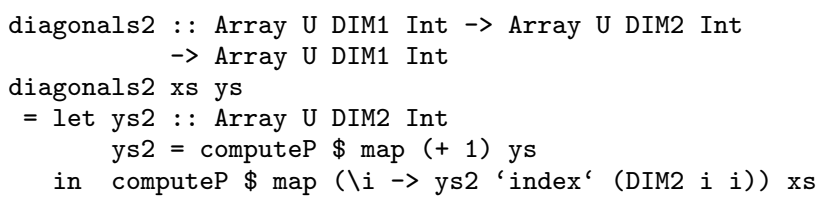

At runtime, the binding for ys 2 involving the first computeP will be suspended by lazy evaluation. This binding will be forced by the second computeP expression when it tries to evaluate the initial element in the overall result of diagonals 2 . When one parallel computation invokes another it is nested parallelism, which Repa does not support. Our current implementation will print a warning to stderr and then run the inner computeP sequentially. 
Although this behaviour provides the expected result at the value level, sequential evaluation is unlikely to be what the user intended - especially because they wrote computeP (with a parallel P). To ensure that both applications of computeP actually run in parallel, evaluation of ys 2 must complete before the second computeP starts. Once again, this can be fixed with a bang pattern:

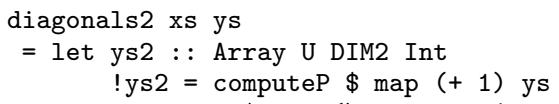

The Repa library is written so that when the first parallel computation is evaluated, it unsafely initialises a globally shared gang of threads (with unsafePerformI0). All subsequent parallel computations run on this single gang of threads, and hence only one can run at a time. We do not create thread gangs dynamically because a single, well balanced data parallel computation is always enough to keep all threads busy. If we had multiple gangs running concurrently, then they would contend for cache and thrash the OS scheduler. Importantly, using an unsafely initialised gang of threads does not violate observational purity (other than on stderr), because all Repa computations still return the correct value, even though nested computations may run sequentially.

Should we change Repa to support slow nested parallel computations that the user probably didn't mean to write? Probably not! Until we have a way to statically guarantee that only one parallel computation runs at a time, we offer the following function in the default API:

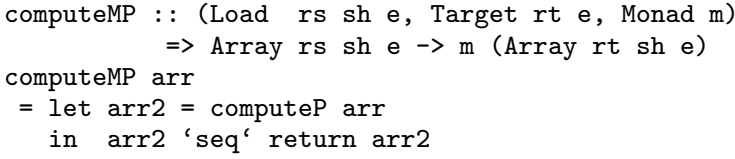

The function computeMP is like computeP, except that it forces completion at a particular point in a monadic computation. Writing diagonals2 with do-notation and using computeMP will achieve the same result as adding the bang pattern to ys 2 . In fact, only computeMP is exposed in the top-level Repa module. The user needs to go looking to find computeP, before they can get themselves in trouble with fake nested parallelism.

Note that computeMP is parametric in the monad as we only need a well defined notion of sequence, rather than a particular monadic effect. Of course, the user could instantiate this to the ST monad and still run two parallel computations concurrently, just like they could instantiate it to IO and use forkIO to achieve the same result. Both of these operations would be considered "safe" in the Haskell development culture. It would be nice if our types could enforce all the desired performance characteristics, but as of now they are only a guide.

\section{Related Work}

For general related work on high-level array libraries, we refer to the relevant discussions in the previous two papers on Repa [5, 8]. Here we only review work on using type indices to guide representations.

The MTLX (Monad Transformer Library indeXed) library [16] uses type indices to allow multiple distinct instances of a monadic effect to coexist in the same computation. For example, a monadic computation can have access to state for a fresh name supply as well as an event counter, and the type of the computation reveals which state objects it accesses.

The C++ parallel array library POOMA [13] uses templates with tags that are like type indices to specify the representation of arrays. Instead of Repa-style delayed arrays, POOMA use expression templates to reify expressions and to facilitate array fusion.
Acknowledgements Thanks to Ben Lambert-Smith for the original Fluid Flow example, Michael Orlitzky for the interpolation example, and Andres Löh for discussion about the role of computeMP. During the development of Repa 1, Roman Leshchinskiy argued that we should distinguish between array representations at the type level - he was right. This work was supported in part by the Australian Research Council under grant number LP0989507.

\section{References}

[1] S. Carr, C. Ding, and P. Sweany. Improving software pipelining with unroll-and-jam. In Hawaii International Conference on System Sciences. IEEE Computer Society, 1996.

[2] M. M. T. Chakravarty, G. Keller, S. L. Peyton Jones, and S. Marlow. Associated types with class. In POPL: Principles of Programming Languages. ACM, 2005.

[3] Coq. The Coq Proof Assistant Reference Manual Version 8.3, 2012. URL http: //coq.inria.fr.

[4] D. J. Jr., S. Marlow, and S. Singh. Parallel performance tuning for Haskell. In Haskell Symposium. ACM, 2009.

[5] G. Keller, M. M. T. Chakravarty, R. Leshchinskiy, S. L. Peyton Jones, and B. Lippmeier. Regular, Shape-polymorphic, Parallel Arrays in Haskell. In ICFP: International Conference on Functional Programming. ACM, 2010.

[6] T.-Y. Lee, C. S. Raghavendra, and J. B. Nicholas. Experimental evaluation of load balancing strategies for ray tracing on parallel processors. In ICPP: International Conference on Parallel Processing. IEEE, 1994.

[7] C. Li, C. Ding, and K. Shen. Quantifying the cost of context switch. In Workshop on Experimental Computer Science. ACM, 2007.

[8] B. Lippmeier and G. Keller. Efficient Parallel Stencil Convolution in Haskell. In Haskell Symposium. ACM, 2011.

[9] S. Marlow, A. R. Yakushev, and S. L. Peyton Jones. Faster laziness using dynamic pointer tagging. In ICFP: International Conference on Functional Programming. ACM, 2007.

[10] U. Norell. Dependently typed programming in Agda. In TLDI: Types in Language Design and Implementation. ACM, 2009.

[11] S. L. Peyton Jones, W. Partain, and A. Santos. Let-floating: Moving bindings to give faster programs. In ICFP: International Conference on Functional Programming. ACM, 1996.

[12] S. L. Peyton Jones, A. Tolmach, and T. Hoare. Playing by the rules: Rewriting as a practical optimsiation technique in GHC. In Haskell Workshop. ACM, 2001.

[13] J. Reynders, J. C. Cummings, P. J. Hinker, M. Tholburn, S. Banerjee, M. Srikant, S. Karmesin, S. Atlas, S. Keahey, and W. F. Humphrey. Pooma: A framework for scientific simulation on parallel architectures. In Parallel Programming using C++. MIT Press, 1996.

[14] B. K. Rosen, M. N. Wegman, and F. K. Zadeck. Global value numbers and redundant computations. In POPL: Principles of Programming Languages. ACM, 1988.

[15] J. Salmon and J. Goldsmith. A hypercube ray-tracer. In Hypercube Concurrent Computers and Applications, C3P. ACM, 1988.

[16] M. Snyder and P. Alexander. Monad factory: Type-indexed monads. In Trends in Functional Programming. Springer, 2010.

[17] T. Sorokina and F. Zeilfelder. Local quasi-interpolation by cubic $C^{1}$ splines on type-6 tetrahedral partitions. IMA Journal of Numerical Analysis, 27(1), 2007.

[18] J. Stam. Stable fluids. In SIGGRAPH, 1999. 